\title{
Several New Data on the Electrical Conduction and Magnetism of Highly Oxidized $\operatorname{SrCoO}_{x}(2.46 \leq x \leq 2.80)$
}

\author{
S. Nakamura, Y. Tadokoro and S. Iida
}

Department of Physics, Teikyo University, 1-1 Toyosatodai, Utsunomiya 320, Japan

\begin{abstract}
The electrical conduction and magnetic properties of highly oxidized polycrystalline $\operatorname{SrCoO}_{x}(2.46 \leq x \leq 2.80)$ were measured down to $10 \mathrm{~K}$. Unlike the previous reports, the temperature coefficient, $d \rho / d T$, of all the specimens are negative. From the magnetic and electrical properties of high temperature phase system (orthorhombic-tetragonal; pseudo-perovskite), the transition from localized nature to band character is suggested for the $3 \mathrm{~d}$ electrons. The tetragonal phases are ferromagnetic. But the low temperature phases are paramagnetic. There may be a correlation between the conductivity and the local ordering of the magnetic spins below around $100 \mathrm{~K}$.
\end{abstract}

\section{INTRODUCTION}

Strontium cobaltate $\mathrm{SrCoO}_{x}$ system is known to exhibit several phases with different electronic states of Co ions introduced by the change in the valency. There are two distinct phases in $\mathrm{SrCoO}_{x}$, i.e., high temperature phase (orthorhombictetragonal-cubic system, or pseudo-perovskite) and low temperature phase (hexagonal system). In early 1970's, Watanabe et al. [1-3] reported that the former system with $x$ close to 3.0 is ferromagnetic metal whereas the latter is paramagnetic metal. But the specimens prepared at that time are supposed to contain $\mathrm{NaCO}_{2} \mathrm{O}_{4}$, which we recently reported to be a good metallic conductor [4]. For the ferromagnetism of the high temperature phase system, they later reported more details by using single crystals $[5,6]$.

We have investigated the electrical conduction and magnetic properties of highly oxidized polycrystalline $\mathrm{SrCoO}_{x}$ $(2.46 \leq x \leq 2.80)$ system down to $10 \mathrm{~K}$ with the high accuracy. Here we report briefly our several new data.

\section{EXPERIMENTAL PROCEDURES}

The specimens were prepared by a conventional ceramic method using $\mathrm{SrCO}_{3}$ (99.9\%) and $\mathrm{Co}_{3} \mathrm{O}_{4}$ (99.9\%) powders as the starting materials. To obtain the high temperature phases, the specimens were initially quenched into liquid nitrogen from $1273 \mathrm{~K}$, while the low temperature phases were obtained by slow cooling in the furnace. Various heat treatments adopted are listed in Table I together with their lattice constants determined by $\mathrm{Cu} \mathrm{K}_{\alpha} \mathrm{X}$ ray diffraction. The orthorhombic $\left(a_{0} \approx \sqrt{2} a_{\mathrm{c}}\right.$, $\left.b_{\mathrm{o}} \approx 4 a_{\mathrm{c}}, c_{\mathrm{o}} \approx \sqrt{2} a_{\mathrm{c}}\right)$ and tetragonal $\left(a_{\mathrm{t}} \approx 2 \sqrt{2} a_{\mathrm{c}}, c_{\mathrm{t}} \approx 2 a_{\mathrm{c}}\right)$ phases are the superstructures resulting from the ordering of oxygen vacancies in the cubic perovskite structure [7]. The subscripts $\mathrm{o}, \mathrm{t}, \mathrm{c}$, and $\mathrm{h}$ indicate orthorhombic, tetragonal, cubic, and hexagonal structures, respectively. The oxygen content, $x$, was determined by the iodometric titration method. The electrical resistivity of the speciznens were measured by a conventional four terminal method (Figs. 1, 2). The static magnetization measurements were carried out by means of a vibrating sample magnetometer ( $\pm 15 \mathrm{kOe}$, Fig. 3 ) and a high field magnetometer installed in a superconducting magnet $( \pm 150 \mathrm{kOe}$, Fig.4).

Table I. The crystal structure of $\mathrm{SrCoO}_{x}$ specimens. Here LQ and SC denote quenching in liquid nitrogen and slow cooling in the furnace, respectively. Subscripts $o, t$, and $h$ indicate orthorhombic, tetragonal, and hexagonal phases, respectively.

\begin{tabular}{|c|c|c|c|}
\hline final heat treatment & composition & crystal structure & lattice constants $(A)$ \\
\hline LQ from $1273 \mathrm{~K}, P_{\mathrm{O}_{2}}=0.2 \mathrm{~atm}$ & $\mathrm{SrCOO}_{2.46}(0)$ & orthorhombic & $a_{0}=5.479, b_{0}=15.719, c_{0}=5.544$ \\
\hline $\mathrm{SC}$ from $673 \mathrm{~K}, \quad P_{\mathrm{O}_{2}}=0.2 \mathrm{~atm}$ & $\mathrm{SrCOO}_{2.52}(\mathrm{o})$ & orthornombic & $a_{0}=5.470, b_{0}=15.715, c_{0}=5.540$ \\
\hline SC from $673 \mathrm{~K}$, & $\mathrm{SrCoO}_{2.70}(\mathrm{t})$ & tetragonal & $a_{\mathrm{t}}=10.91, c_{\mathrm{t}}=7.708$ \\
\hline SC from $673 \mathrm{~K}$ & $\mathrm{SrCOO}_{2.78}(\mathrm{t})$ & tetragonal & $a_{\mathrm{t}}=10.910, c_{\mathrm{t}}=7.691$ \\
\hline SC from $673 \mathrm{~K}$ & $\mathrm{SrCoO}_{2.80}(\mathrm{t})$ & tetragonal & $a_{\mathrm{t}}=10.90, c_{\mathrm{t}}=7.61$ \\
\hline LQ from $973 \mathrm{~K}$ & $\mathrm{SrCoO}_{2.60}(\mathrm{~h})$ & hexagonal & $a_{\mathrm{h}}=5.497, c_{\mathrm{h}}=4.162$ \\
\hline $\mathrm{SC}$ from $1273 \mathrm{~K}, P_{\mathrm{O} 2}=0.2 \mathrm{~atm}$ & $\mathrm{SrCoO}_{2.73}$ (h) & hexagonal & $a_{\mathrm{h}}=5.491, c_{\mathrm{h}}=4.145$ \\
\hline $\mathrm{SC}$ from $673 \mathrm{~K}, \quad P_{\mathrm{O}_{2}}=40 \mathrm{~atm}$ & $\mathrm{SrCoO}_{2.75}$ (h) & hexagonal & $a_{\mathrm{h}}=5.488, c_{\mathrm{h}}=4.132$ \\
\hline
\end{tabular}




\section{RESULTS AND DISCUSSION}

As shown in Figs. 1 and 2, the resistivity $(\rho)$ decreases with increasing $x$, i.e., the oxygen content, which was not clear in the previous reports $[1,3]$. The resistivity reaches to the order of $10^{-1} \Omega \mathrm{cm}$ at room temperature for $S r C o O_{2.80}(t)$, but unlike the previous reports, all specimens show negative temperature coefficients ( $d \rho d \mathrm{~d} T$ ) at all temperatures. In addition, the $\rho-T$ curves of the hexagonal specimens show a flat portions at about $50-100 \mathrm{~K}$.

As shown in Fig. 3, the tetragonal phases are ferromagnets with $T_{\mathrm{c}} \approx 290 \mathrm{~K}$. As shown in Fig. 4, the magnetization curves at $4.2 \mathrm{~K}$ have large hysteresis up to $60-80 \mathrm{kOe}$ and the magnetization $(\sigma)$ do not completely saturate even at $150 \mathrm{kOe}$. Therefore anomalies around $50 \mathrm{~K}$ in the thermomagnetic curves of Fig. 3 are due to the large magnetic anisotropy. The magnetic moments at $4.2 \mathrm{~K}$ are estimated to be $1.1 \mu_{\mathrm{B}}, 0.64 \mu_{\mathrm{B}}$, and $0.67 \mu_{\mathrm{B}}$ per Co ion for $x=2.70(\mathrm{t}), 2.78(\mathrm{t})$, and 2.80 $(t)$, respectively. The large moment of $\mathrm{SrCoO}_{2.70}(\mathrm{t})$ is new. Assuming that all the 3d electrons of Co ion occupy de band, which is equivalent to the low spin configuration, the magnetic moments calculated are $0.4 \mu_{\mathrm{B}}, 0.56 \mu_{\mathrm{B}}$, and $0.6 \mu_{\mathrm{B}}$, respectively. This simple band model seems effective for $x=2.78(t)$ and $2.80(t)$, but not for $x=2.70(t)$. It is expected that $\mathrm{SrCoO}_{2.70}(\mathrm{t})$, which is not so conductive as the other two (Fig.1), will have its 3d electrons still localized nature and partially in high spin or intermediate spin configuration. This will be supported by the fact that the pseudo-perovskite $\mathrm{SrCoO}_{2.50}(0)$ is an antiferromagnet $\left(T_{\mathrm{N}}=570 \mathrm{~K}\right)$ with high spin configuration [2].

On the other hand, the hexagonal phases are paramagnetic, as shown in Fig. 5. The magnetic susceptibility $(\chi)$ decreases with increasing $x$. The $\chi$-T curves seem to follow Curie-Weiss law above around $100 \mathrm{~K}$, indicating the effective magnetic moments of about $2.3 \mu_{\mathrm{B}}$ per Co ion for all the three specimens. This value is between those of low and high spin state. Below around $100 \mathrm{~K}$, the $\chi-T$ curves deviate from the Curie-Weiss law, which may indicate the generation of local spin ordering. The change of the $\rho-T$ curves below around this temperature, shown in Fig. 2, may be due to the decrease in the scattering of conduction electrons by $3 d$ spins. Further investigation is going on to clarify this point.

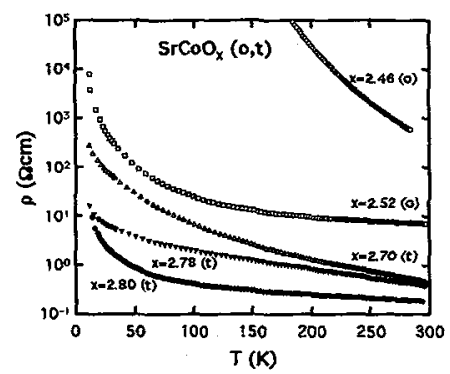

Fig. 1 The $\rho-T$ curves of $\mathrm{SrCOO}_{x}(0, \mathrm{t})$.

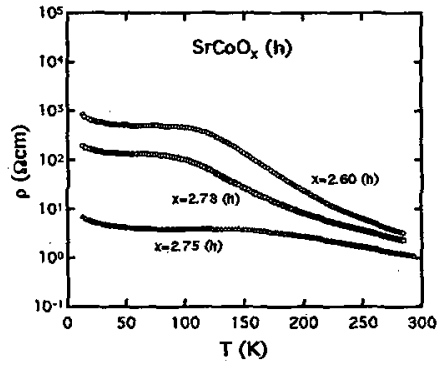

Fig. 2 The $\rho-T$ curves of $\mathrm{SrCoO}_{x}(\mathrm{~h})$.

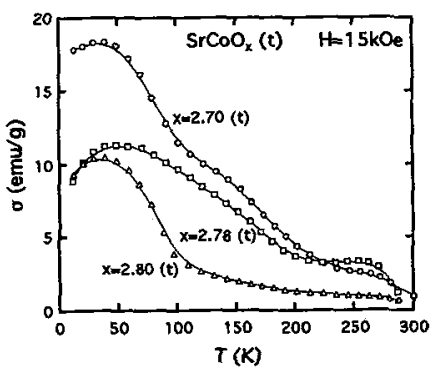

Fig. 3 The $\sigma-T$ curves of $\mathrm{SrCoO}_{x}(\mathrm{t})$.

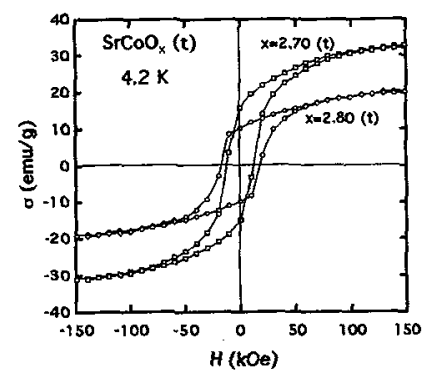

Fig. 4 The $\sigma-H$ curves of $\mathrm{SrCoO}_{x}(t)$ at $4.2 \mathrm{~K}$

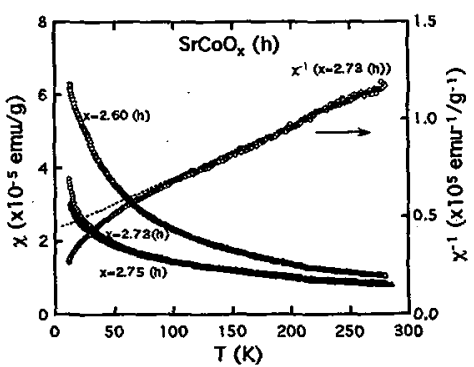

Fig. 5 The $\chi-T$ and $\chi^{-1}-T$ curves of $\mathrm{SOCOO}_{x}(\mathrm{~h})$.

\section{Acknowledgment}

The authors are grateful to Mr. I. Oguro. Institute for Solid State Physics, University of Tokyo, for the high field magnetization measurements.

\section{References}

[1] Watanabe H. and Takeda T., Proc. Intl. Conf. on Ferrites (Univ. of Tokyo Press, Tokyo, 1971) pp. 588-590.

[2] Takeda T., Yamaguchi Y. and Watanabe H., J.Phys. Soc. Jpn. 33 (1972) 970-972.

[3] Takeda T. and Watanabe H., J.Phys. Soc. Jpn. 33 (1972) 973-978.

[4] Tanaka T., Nakamura S. and Lida S., JpnJ JAppl.Phys. 33 (1994), L581-L582.

[5] Takei H., Oda H., Watanabe H. and Shindo I., J. Mater. Sci. 13 (1978) 519-522.

[6] Watanabe H., Yamaguchi Y., Oda H. and Takei H., J. Magn. Magn. Mat. 15-18 (1980) 521-522.

[7] Takeda Y., Kanno R., Takada T., Yamamoto O., Takano M. and Bando Y., Z. anorg. allg. Chem. $540 / 541$ (1986) 259-270. 